\title{
Excitable CO Oxidation on Pt(110) under Nonuniform Coupling
}

\author{
C. Beta, M. G. Moula, A. S. Mikhailov, H. H. Rotermund, and G. Ertl \\ Fritz-Haber-Institut der Max-Planck-Gesellschaft, Faradayweg 4-6, 14195 Berlin, Germany
}

(Received 25 June 2004; published 28 October 2004)

\begin{abstract}
A new feedback scheme for guided spatiotemporal pattern formation in reaction-diffusion systems is introduced. In contrast to previously established control methods, we present a coupling protocol that is sensitive to the presence of coherent structures in the medium. Applying this feedback to the catalytic $\mathrm{CO}$ oxidation on $\operatorname{Pt}(110)$ in both experiments and numerical simulations, we show that temporal evolution and spatial extension of self-organizing objects can be efficiently controlled.
\end{abstract}

DOI: 10.1103/PhysRevLett.93.188302

The study of pattern formation in extended nonlinear media has grown into a rapidly developing interdisciplinary domain of research [1]. Recent contributions in this field focus on control of pattern formation processes and aim at the generation of novel patterns. Much of this work was performed with spatially distributed systems showing oscillatory dynamics of their individual elements. In such systems, complex spatiotemporal patterns could be generated and stabilized by application of external periodic forces or simple feedback loops [2-4]. In excitable media, previous efforts were directed at controlling the behavior of individual coherent structures that could be considered as basic building blocks of more complex spatiotemporal patterns. By introducing global feedback, traveling spots near the onset of translational motion could be stabilized [5] and rotating spiral waves in confined geometries were manipulated [6]. In experiments with the photosensitive Belousov-Zhabotinsky reaction, stabilization of traveling wave fragments was achieved by globally controlling the excitability of the medium [7] and complex individual and collective motion of such fragments in the presence of spatial excitability gradients could be observed [8]. For surface chemical reactions, individual addressing and modification of traveling waves through local heating by means of focused laser beams was recently demonstrated [9]. Engineering of chemical complexity, including selective creation and controlled evolution of coherent structures is the ultimate goal of these efforts.

In this Letter, we propose a novel feedback scheme that constitutes a step towards the design of more advanced methods for the control of pattern formation in excitable media. A feedback loop is introduced that is sensitive to the length scales of patterns emerging in the medium. In a chemical reaction-diffusion system, this type of control can be implemented in the following way,

$$
\begin{aligned}
& \partial_{t} \mathbf{c}=\mathbf{f}[\mathbf{c}, q(t)]+\mathbf{D} \nabla^{2} \mathbf{c}, \\
& q(t)=q_{0}+\mu\left|\hat{c}_{i}^{\mathbf{k}}(t-\tau)\right|,
\end{aligned}
$$

with $\hat{c}_{i}^{\mathbf{k}}=\frac{1}{S} \int_{S} e^{-\mathrm{i} \mathbf{k r}} c_{i}(\mathbf{r}, t) \mathrm{d} \mathbf{r}$. Here, $\mathbf{c}=\left(c_{1}, c_{2}, \ldots, c_{N}\right)$ are the concentrations of reacting species with the diffu-
PACS numbers: 82.40.Np, 05.45.-a, 47.54.+r, 82.40.Ck

sion matrix $\mathbf{D}$. The nonlinear functions $\mathbf{f}(\mathbf{c}, q)$ account for the reaction kinetics and depend on some global parameter $q$ like temperature or pressure. The feedback signal is computed from the spatial distribution of one of the reacting components, $c_{i}$. It is chosen proportional to the modulus of a Fourier coefficient from the spatial spectrum of the distribution of $c_{i}$ and thus selectively depends on the length scales of the patterns in the system. The Fourier coefficient is determined by fixing the corresponding wave vector $\mathbf{k}$, which is a parameter in the coupling scheme. For $k \equiv|\mathbf{k}| \neq 0$, a feedback signal is generated only if spatially nonuniform structures are present in the medium. Therefore, in contrast to feedback signals that are computed from the spatial average of a system variable $(k=0)$, the present coupling method does not ultimately drive the system to a uniform state but is expected to stabilize, within a large interval of feedback intensities $\mu$, a situation where spatial patterns prevail. In the following, we will refer to this feedback as nonuniform coupling since it is sensitive to the presence of spatially nonuniform structures.

Among the class of pattern forming heterogeneous catalytic reactions [10] the oxidation of $\mathrm{CO}$ on a $\mathrm{Pt}(110)$ surface is the most thoroughly studied example. Besides the Belousov-Zhabotinsky reaction, the catalytic $\mathrm{CO}$ oxidation on platinum surfaces was established as one of the few accessible systems where the nonlinear dynamics of a pattern forming medium can be studied experimentally [11]. In the present work, we apply the above introduced nonuniform coupling to the catalytic $\mathrm{CO}$ oxidation on $\operatorname{Pt}(110)$ in both experiments and numerical simulations.

Experimental.-The experiments were carried out under low pressure conditions on a $\mathrm{Pt}(110)$ single crystal with a diameter of about $10 \mathrm{~mm}$ mounted inside an ultrahigh vacuum chamber. The oxidation of $\mathrm{CO}$ proceeds through a Langmuir-Hinshelwood mechanism. Both $\mathrm{CO}$ and oxygen have to adsorb on the catalytic surface before $\mathrm{CO}_{2}$ can be formed which is immediately released into the gas phase [12]. Together with an adsorbate dependent structural change of the catalyst surface, the interplay of reaction and surface diffusion of $\mathrm{CO}$ allows the emergence of a wealth of complex spatiotemporal 
adsorbate patterns on the platinum surface [11]. Exploiting the adsorbate dependent change in local work function, we use a photoemission electron microscope (PEEM) to obtain spatially resolved real time images of the concentration patterns on the catalyst [13]. Because of the difference in work function between $\mathrm{O}$ and $\mathrm{CO}$ covered platinum, we can identify $\mathrm{CO}(\mathrm{O})$ covered areas as bright (dark) regions in the PEEM image. Anisotropy of CO diffusion on the $\mathrm{Pt}(110)$ surface induces an elliptical elongation of curved structures.

Oxygen treatment at $600 \mathrm{~K}$, repeated cycles of argon ion sputtering, and annealing up to $1000 \mathrm{~K}$ were carried out to prepare the sample surface. Temperature was maintained by a halogen lamp back side heating and the partial pressures could be controlled by an electronically operated dosing system. Into this setting, we implemented a feedback loop according to Eqs. (1) and (2). At each instant, a two-dimensional fast Fourier transform is applied to the PEEM image $I(x, y)$. The feedback signal is then computed from the modulus of a Fourier coefficient $\hat{I}^{\mathbf{k}}$ in the frequency plane, the wave vector $\mathbf{k}=\left(k_{x}, k_{y}\right)$ being a feedback parameter. We averaged the value of the Fourier coefficient over a $3 \times 3$ pixel region at the tip of the wave vector. The elliptical shape of the concentration patterns on the $\operatorname{Pt}(110)$ surface is reflected by an anisotropic frequency pattern in the $\left(k_{x}, k_{y}\right)$ plane. However, we restricted our choice of $\mathbf{k}$ to a single direction (the diagonal of the upper right quadrant of the frequency plane, $k_{x}=k_{y}$ and $k_{x}, k_{y}>0$ ), so that $\hat{I}^{\mathbf{k}}$ is uniquely defined by fixing $k=|\mathbf{k}|=\sqrt{k_{x}^{2}+k_{y}^{2}}$ and anisotropy does not have to be considered here. The feedback signal is scaled by an

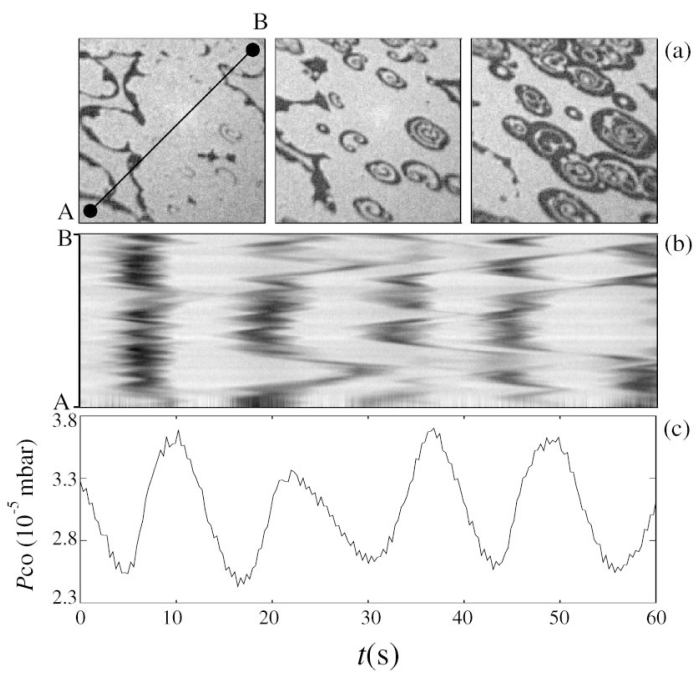

FIG. 1. Pattern formation in the presence of nonuniform coupling, experimental results. (a) PEEM images of typical patterns during one evolution cycle. (b) Space-time diagram along the diagonal $A B$ indicated in the first snapshot in (a). (c) Evolution of $\mathrm{CO}$ partial pressure in the reactor. The parameters are $T=453 \mathrm{~K}, p_{\mathrm{O}_{2}}=4 \times 10^{-4}$ mbar, $p_{\mathrm{CO}}^{0}=2.28 \times$ $10^{-5}$ mbar, $\mu=1.54 \times 10^{-5} \mathrm{mbar}, k=17.0$, and $\tau_{\mathrm{ext}}=0 \mathrm{~s}$.

$188302-2$ intensity factor $\mu$ and coupled back to the system as a modulation of the $\mathrm{CO}$ partial pressure in the reactor,

$$
p_{\mathrm{CO}}(t)=p_{\mathrm{CO}}^{0}+\mu\left|\hat{I}^{k}(t-\tau)\right|,
$$

where $p_{\mathrm{CO}}^{0}$ is the base $\mathrm{CO}$ partial pressure and $\tau$ a possible control loop latency. Note that the delay $\tau=\tau_{\min }+\tau_{\text {ext }}$ cannot be reduced beyond a minimal intrinsic delay $\tau_{\min }$, which is determined by the finite pumping rate of the reactor and was of the order of $1 \mathrm{~s}$ in our case.

Experiments were carried out in the excitable regime. Initiated at surface defects, oxygen patterns start to develop on a mostly $\mathrm{CO}$ covered surface. If the feedback is set into action, a cyclic behavior is rapidly established in the system: bubble shaped oxygen islands repeatedly grow and get suppressed with a period of the order of 10 s. Figure 1(a) presents a series of three consecutive PEEM images showing typical concentration patterns emerging during one such cycle. In Fig. 1(b), a spacetime diagram is displayed, taken along the line $A B$ indicated in the first snapshot of Fig. 1(a). The alternating dark and light zones clearly reflect the oscillating evolution of patterns on the catalyst. The corresponding evolution of $\mathrm{CO}$ partial pressure is plotted in Fig. 1(c).

In a series of experiments, we studied the overall behavior of these pattern formation cycles by systematic variation of the feedback intensity $\mu$ and the delay time $\tau_{\text {ext }}$. Figure 2(a) shows that the period $T$ of oscillations in $\mathrm{CO}$ partial pressure decreases with increasing feedback intensity $\mu$. However, for large $\mu$ this effect becomes less pronounced and the period levels off at some minimal value. For variation of the delay time $\tau_{\text {ext }}$ we find an
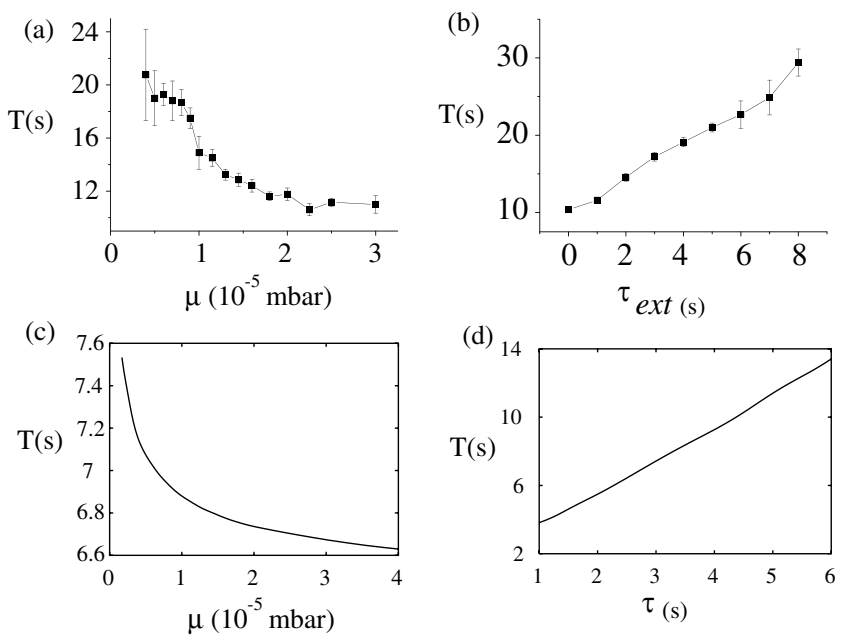

FIG. 2. Period of pattern formation cycles as a function of the feedback intensity $\mu$ (a),(c) and the delay time $\tau$ (b),(d). (Top) Experimental results, (a) $T=473 \mathrm{~K}, \quad p_{\mathrm{O}_{2}}=4 \times$ $10^{-4} \mathrm{mbar}, \quad p_{\mathrm{CO}}^{0}=4.33 \times 10^{-5} \mathrm{mbar}, k_{x}, k_{y}=10, \tau_{\mathrm{ext}}=1 \mathrm{~s}$; (b) $T=449 \mathrm{~K}, p_{\mathrm{O}_{2}}=4 \times 10^{-4} \mathrm{mbar}, p_{\mathrm{CO}}^{0}=2.28 \times 10^{-5} \mathrm{mbar}$, $k_{x}, k_{y}=6, \mu=1.54 \times 10^{-5}$ mbar. (Bottom) Numerical simulations, (c) $\tau=3 \mathrm{~s}$; (d) $\mu=2 \times 10^{-4} \mathrm{mbar}$; and (c), (d) $k=10$. For other parameters, see caption of Fig. 4 . 
opposite effect. As $\tau_{\text {ext }}$ is increased, the period of oscillations grows monotonously, see Fig. 2(b).

Moreover, the characteristic size of the patterns depends on the modulus $k$ of the wave vector. Figure 3(a)3(d) shows typical localized structures for different values of $k$. These oxygen patterns periodically grow and get erased by extending $\mathrm{CO}$ covered areas from the inside. For comparison of the different measurements, we have considered images at equivalent points of such pattern formation cycles. Therefore, the snapshots displayed in Fig. 3(a)-3(d) are taken when the feedback signal reaches its maximum. Obviously, the characteristic size of oxygen islands grows with increasing $k$ from Fig. 3(a)-3(d). Also, the period of oscillations in $\mathrm{CO}$ partial pressure grows for larger values of $k$, see Fig. 3(e).

Modeling.-We performed numerical simulations using the Krischer-Eiswirth-Ertl model [14] — a realistic reaction model that has been thoroughly studied and widely used to model the dynamics of catalytic CO oxidation on $\mathrm{Pt}(110)$. It consists of three coupled partial differential equations,

$$
\begin{gathered}
\partial_{t} u=k_{1} p_{\mathrm{CO}} s_{\mathrm{CO}}\left(1-u^{3}\right)-k_{2} u-k_{3} u v+D \nabla^{2} u, \\
\partial_{t} v=k_{4} p_{\mathrm{O}_{2}}\left[s_{\mathrm{O}, 1 \times 1} w+s_{\mathrm{O}, 1 \times 2}(1-w)\right](1-u-v)^{2}-k_{3} u v,
\end{gathered}
$$

$$
\partial_{t} w=k_{5}\left(\frac{1}{1+\exp \left(\frac{u_{0}-u}{\delta u}\right)}-w\right)
$$

where $u, v$, and $w$ denote the CO coverage, the $\mathrm{O}$ coverage, and the local fraction of the catalyst surface in the

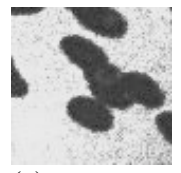

(a)

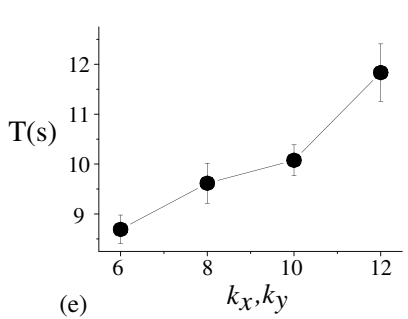

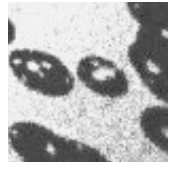

(b)

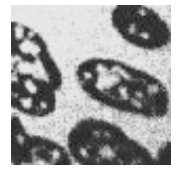

(c)

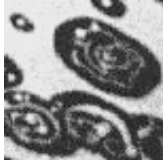

(d)

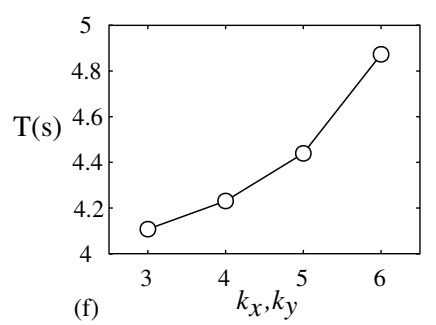

FIG. 3. (Top) Pattern size for varying length $k$ of the wave vector, $k_{x}, k_{y}=6 \quad$ (a), 8 (b), 10 (c), and 12 (d). (Bottom) Dependence of the period of CO partial pressure oscillations on $k$. Experimental parameters for (a)-(d) as in Fig. 1 , (e) $T=449 \mathrm{~K}, \quad p_{\mathrm{O}_{2}}=4 \times 10^{-4} \mathrm{mbar}, p_{\mathrm{CO}}^{0}=2.76 \times$ $10^{-5}$ mbar, $\mu=1.66 \times 10^{-5}$ mbar, $\tau_{\text {ext }}=0 \mathrm{~s}$. (f) Numerical parameters: 2D system $L^{2}$ with $L=400 \mu \mathrm{m}$ and periodic boundaries, $s_{\mathrm{O}, 1 \times 1 / 1 \times 2}$ are increased by 0.04 in a region of $3 \times$ 3 grid points at the center, $\mu / p_{\mathrm{CO}}^{0}=0.06, \tau=1 \mathrm{~s}$, and other parameters as in Fig. 4. nonreconstructed $1 \times 1$ phase, respectively. The equations account for adsorption of $\mathrm{CO}$ and oxygen, reaction between the adsorbed species, desorption and surface diffusion of $\mathrm{CO}$, and the adsorbate dependent structural transition of the $\operatorname{Pt}(110)$ surface. Here, we extend Eqs. (4)-(6) by introducing a feedback mimicking the experiment: the $\mathrm{CO}$ partial pressure $p_{\mathrm{CO}}$ is changing in time depending on the modulus of a chosen Fourier coefficient from the current frequency spectrum of the CO coverage,

$$
p_{\mathrm{CO}}(t)=p_{\mathrm{CO}}^{0}+\mu\left|\hat{u}^{k}(t-\tau)\right| .
$$

Modeling of the image intensity $I$ by the CO coverage $u$ has been previously established in similar configurations, see, e.g., Ref. [4]. Simulations are performed in the excitable regime (for parameters and numerical details see caption of Fig. 4). Inspired by STM data that shows a strain induced enhancement of dissociative adsorption in the vicinity of surface defects $[15,16]$, we model the presence of defects by a locally increased oxygen sticking coefficient. In the one-dimensional simulations, the coefficients $s_{\mathrm{O}, 1 \times 1 / 1 \times 2}$ were increased at a number of randomly chosen locations ( $20 \%$ of the grid points) by a fixed value of 0.02 on average.

Figure 4 shows the space-time diagram (a) and the evolution of $\mathrm{CO}$ partial pressure (b) of a typical model simulation in the presence of nonuniform coupling. We find a cyclic behavior qualitatively similar to the experimental results: starting from surface heterogeneities, patterns repeatedly emerge and get suppressed by the feedback. The period of oscillations in CO partial pressure is of the same order as in the experiment.

We find a good qualitative agreement with the experiment also for the dependence of the period $T$ of pattern

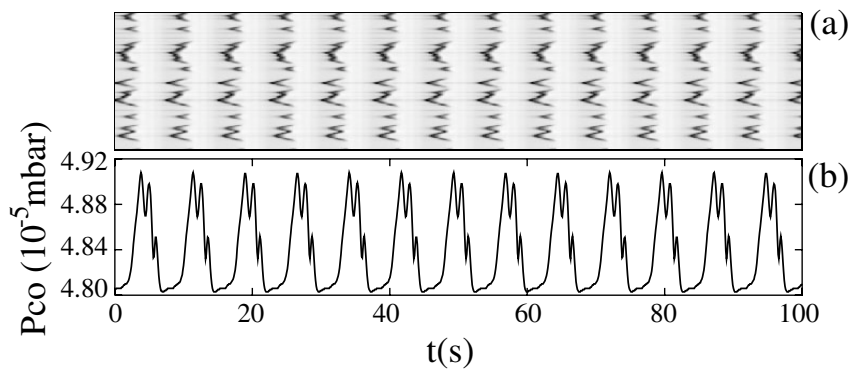

FIG. 4. Pattern formation in the presence of nonuniform coupling, numerical results. (a) Space-time diagram and (b) evolution of $\mathrm{CO}$ partial pressure. Model parameters: $k_{1}=$ $3.14 \times 10^{5} \mathrm{~s}^{-1} \mathrm{mbar}^{-1}, k_{2}=10.23 \mathrm{~s}^{-1}, k_{3}=283.9 \mathrm{~s}^{-1}, k_{4}=$ $5.86 \times 10^{5} \mathrm{~s}^{-1} \mathrm{mbar}^{-1}, \quad k_{5}=1.61 \mathrm{~s}^{-1}, \quad D=40 \mu \mathrm{m}^{2} \mathrm{~s}^{-1}$, $s_{\mathrm{CO}}=1.0, s_{\mathrm{O}, 1 \times 1}=0.6, s_{\mathrm{O}, 1 \times 2}=0.4, u_{0}=0.35, \delta u=0.05$, $p_{\mathrm{CO}}^{0}=4.8 \times 10^{-5} \mathrm{mbar}, p_{\mathrm{O}_{2}}=1.2 \times 10^{-4} \mathrm{mbar}$, and $T=$ $543.5 \mathrm{~K}$. Feedback parameters: $\mu / p_{\mathrm{CO}}^{0}=0.01, k=10$, and $\tau=3 \mathrm{~s}$. Numerics: 1D system of length $L=800 \mu \mathrm{m}$ with periodic boundaries, second-order finite differences with $\Delta x=$ $4 \mu \mathrm{m}$, and an explicit Euler scheme with $\Delta t=0.001 \mathrm{~s}$ are used for integration. 
formation cycles on the feedback parameters. Figs. 2(c), 2(d), and 3(f) show numerical results of systematic scans of the feedback intensity, the delay time, and the wave number $k$ corresponding to the experimental results in Figs. 2(a), 2(b), and 3(e). As in the experiment, the period of oscillations is reduced for increasing feedback intensity $\mu$ and grows with increasing control loop latency $\tau$ and with increasing wavenumber $k$.

Discussion.-In previous approaches to control pattern formation in excitable systems, coupling signals were computed from an averaged system variable $[5,6]$. With respect to these methods, nonuniform coupling can be regarded as a more general form that comprises the earlier work as the particular situation where $k=0$. In the case considered here $(k \neq 0)$, the feedback is sensitive to the presence of spatially nonuniform structures and becomes active only if the system shows patterns that contain the respective mode in their frequency spectrum.

In the parameter regime of the above experiments, we observe an irregular distribution of oxygen pulses and wave fragments, triggered at surface defects and moving on an otherwise CO covered surface. Since in general these objects do not form a regular periodic structure, the frequency spectrum exhibits a broad band of active modes. If $k$ is chosen to be roughly of the same order as the overall length scale of the patterns it can be expected to lie within this band of active modes. Thus, if patterns emerge in the system, the coupling will respond with an increase in $\mathrm{CO}$ partial pressure which counteracts the further growth of oxygen patterns and, therefore, can be regarded as a negative feedback. It induces a periodic behavior of the reaction depending, of course, on the delay in the control loop: oxygen patterns emerge, the feedback becomes active, and the $\mathrm{CO}$ partial pressure increases, so that the patterns get suppressed, the feedback signal vanishes, the $\mathrm{CO}$ pressure drops, and again patterns can start to emerge.

This scenario offers a rationalization of the results shown in Figs. 2 and 3. For larger feedback intensities $\mu$ the emergence of patterns leads to a steeper increase in $\mathrm{CO}$ partial pressure. Patterns then get erased more rapidly and the period of the cyclic pattern evolution becomes shorter as was found in Fig. 2(a) and 2(c). However, for large $\mu$ the feedback signal almost instantaneously crosses the $p_{\mathrm{CO}}$ level where oxygen patterns get suppressed so that a continuing increase in $\mu$ does not shorten the response time of the coupling scheme any further and the period converges to a minimum value. An increasing delay in the feedback loop simply extends the interval between the emergence of patterns and the response of the coupling scheme so that the overall period of the oscillatory cycles grows linearly as shown in Fig. 2(b) and 2(d). Within some interval of $k$ values, both experiments and numerical simulations show a growing period for increasing $k$, see Fig. 3(e) and 3(f).
Within this range, the coefficients in the frequency spectrum are decaying for larger $k$, which corresponds to a decreasing feedback intensity and is thus consistent with the results shown in Fig. 2(a) and 2(c). Since the final size of the oxygen islands depends on the time interval during which they can grow, larger structures are observed for longer periods $T$ and hence for increasing $k$.

In summary, we have introduced nonuniform coupling as a new type of feedback and presented first results from its application to an experimental reaction-diffusion system, the catalytic oxidation of $\mathrm{CO}$ on $\mathrm{Pt}(110)$. The experimental results are supported by numerical simulations performed in the framework of a well established kinetic model for this reaction. Nonuniform coupling can be regarded as a first step along the road to flexible feedback protocols for guided self-organization in nonequilibrium systems. Future work will be directed at tailoring new coupling algorithms to fulfill specific tasks by incorporating other signal transforms and tools for pattern recognition.

We acknowledge financial support of the Deutsche Forschungsgemeinschaft (SFB 555) and of the Humboldt Stiftung.

[1] M. C. Cross and P. C. Hohenberg, Rev. Mod. Phys. 65, 851 (1993).

[2] V. Petrov, Q. Ouyang, and H. L. Swinney, Nature (London) 388, 655 (1997).

[3] V. K. Vanag, L. Yang, M. Dolnik, A. M. Zhabotinsky, and I. R. Epstein, Nature (London) 406, 389 (2000).

[4] M. Kim, M. Bertram, M. Pollmann, A. v. Oertzen, A. S. Mikhailov, H. H. Rotermund, and G. Ertl, Science 292, 1357 (2001).

[5] K. Krischer and A. Mikhailov, Phys. Rev. Lett. 73, 3165 (1994).

[6] V. S. Zykov, A. S. Mikhailov, and S. C. Müller, Phys. Rev. Lett. 78, 3398 (1997).

[7] E. Mihaliuk, T. Sakurai, F. Chirila, and K. Showalter, Phys. Rev. E 65, 065602 (2002).

[8] T. Sakurai, E. Mihaliuk, F. Chirila, and K. Showalter, Science 296, 2009 (2002).

[9] J. Wolff, A. G. Papathanasiou, I. G. Kevrekidis, H. H. Rotermund, and G. Ertl, Science 294, 134 (2001).

[10] R. Imbihl and G. Ertl, Chem. Rev. 95, 697 (1995).

[11] S. Jakubith, H. H. Rotermund, W. Engel, A. von Oertzen, and G. Ertl, Phys. Rev. Lett. 65, 3013 (1990).

[12] T. Engel and G. Ertl, Adv. Catal. 28, 1 (1979).

[13] H. H. Rotermund, J. Electron Spectrosc. Relat. Phenom. 99, 41 (1999).

[14] K. Krischer, M. Eiswirth, and G. Ertl, J. Chem. Phys. 96, 9161 (1992)

[15] M. Gsell, P. Jakob, and D. Menzel, Science 280, 717 (1998).

[16] J. Wintterlin, T. Zambelli, J. Trost, J. Greeley, and M. Mavrikakis, Angew. Chem., Int. Ed. Engl. 42, 2850 (2003). 